\title{
Autoantibodies to Oxidized Low-Density Lipoprotein in Patients with Aortic Regurgitation: Association with Aortic Diameter Size
}

\author{
Sara Shimoni Iris Bar Liaz Zilberman Jacob George \\ The Heart Institute, Kaplan Medical Center, Rehovot, Israel
}

\section{Key Words}

Anti-oxidized low-density lipoprotein antibodies .

Aortic regurgitation - Aortic dilatation

\begin{abstract}
Background: Aortic regurgitation (AR) is a condition associated with volume overload, causing left-ventricular (LV) remodeling, eccentric LV hypertrophy and eventually heart failure. LV remodeling associated with AR is regulated by mechanical stress, neurohormonal activation, inflammation and oxidative stress. Since anti-oxidized low-density lipoprotein (LDL) antibodies (Abs) are a measurable marker of oxidative stress, we hypothesized that an increased level of circulating oxidized LDL (oxLDL) Abs may be related to remodeling of the left ventricle in patients with significant AR. Methods: We assessed IgG anti-oxLDL Abs in 31 patients with significant $A R$ and compared them to 30 patients with similar risk factors and no valvular disease. Abs to oxLDL were determined by ELISA. Results: The 2 groups had similar clinical characteristics. There was no difference between patients with AR and patients with no AR in the level of antioxLDL Abs. However, in all patients and controls, anti-oxLDL Abs correlated positively with the diameter of the ascending aorta ( $A A ; r=0.32, p=0.016$ ) and the level of oxLDL Abs was significantly higher in patients with an AA diameter $\geq 39 \mathrm{~mm}$. On multivariate analysis, only white blood cell count and $A A$ diameter were related to anti-oxLDL Abs in all patients. Conclusions: We did not find a difference in the level of anti-
\end{abstract}

oxLDL Abs between patients with AR and controls; however, there was a strong correlation between anti-oxLDL Abs and AA diameter.

(c) 2014 S. Karger AG, Basel

\section{Introduction}

Oxidized low-density lipoprotein (oxLDL) is present in the plasma and correlates with the extent of atherosclerosis [1,2]. It is also found in calcified aortic valves (AVs) and degenerative aortic bioprosthesis $[3,4]$. oxLDL is a biologically active molecule and is a potent proinflammatory agent in the arterial wall. It is highly immunogenic as reflected in the presence of anti-oxLDL antibodies (Abs) in the circulation as well as in atherosclerotic lesions [5]. It has been shown that the association of oxLDL levels and coronary artery disease (CAD) is superior to other biochemical markers, such as total cholesterol, triglycerides, apolipoprotein B or high-density lipoprotein (HDL) levels [6]. In addition, oxidation leads to an array of potential forms, which appear at different stages of the oxidation process and vary significantly [7]. However, the direct measurement of oxLDL in serum or plasma is complicated by the potential for in vivo modification of the

The Heart Institute is affiliated to the Hebrew University and Hadassah Medical School, Jerusalem, Israel.

\section{KARGER}

E-Mail karger@karger.com

www.karger.com/crd
(C) 2014 S. Karger AG, Basel

$0008-6312 / 14 / 1281-0054 \$ 39.50 / 0$
Sara Shimoni, MD

Kaplan Medical Center POB 1

Rehovot 76400 (Israel)

E-Mail Sara_s@clalit.org.il 
sample and also by the possibility that the primary location of the analyses of interest may not be in the circulation. The measurement of Abs to oxLDL are more likely to accurately reflect a state of heightened oxidative stress present over an extended period as the generation of IgG Abs is a relatively long process whereas oxidative modification occurs within seconds and is amenable to multiple flaws due to inadequate or delayed sample collection.

Abs to oxLDL are detected in human sera, although their biological significance is not well established. The relation between oxLDL and anti-oxLDL Abs is controversial. An inverse relationship has been shown between IgM anti-oxLDL Abs and oxLDL levels and these Abs appear to be protective against atherosclerosis [8]. However, IgG anti-oxLDL Abs are related to atherosclerosis, as is oxLDL. Assessment of oxLDL Abs may reflect the level of oxidative stress and indeed, oxLDL Abs have been shown to correlate with the extent of coronary, carotid and peripheral atherosclerosis and predict future myocardial infarction [9-13].

Aortic regurgitation (AR) is characterized by the diastolic reflux of blood from the aorta into the left ventricle due to malcoaptation of the aortic cusps [14]. AR produces an increased stroke volume, a high pulse pressure and a diastolic retrograde flow that extends to the distal aorta, resulting in volume overload, eccentric left ventricular (LV) hypertrophy and, eventually, heart failure. Previous reports studied the correlation between AR and atherosclerosis. Kervancioglu et al. [15] found more carotid and brachial artery atherosclerosis in patients with AR than in matched controls. Su et al. [16] showed that carotid atherosclerosis is associated with increased pulsepressure and AR; however, this relationship was attenuated after controlling for age. We recently observed significant descending aortic atherosclerosis in patients with severe $A R$, independent of the traditional risk factors and etiology of AR [17]. We have also shown that, similar to in atherosclerosis, patients with AR have decreased numbers of endothelial progenitor cells [18].

Ascending aorta (AA) dilatation is common in patients with AR. Aortic aneurysm may be the primary cause of AR or else it is due to the same pathology as seen in bicsuspid AV and is secondary to an increased and turbulent flow. Thoracic aortic aneurysm is a chronic inflammatory disease. We reason that aortic root dilatation may cause significant hemodynamic changes due to turbulence and the proximity to aortic leaflets. This shear stress imposed on the vessel wall during years of continuous turbulence is known to trigger the upregulation of endothelial cell adhesion molecules that mirror endothe- lial dysfunction, which consequently acts to modify LDL in several ways, the predominant of which is oxidation.

We therefore hypothesized that an increased level of circulating oxLDL Abs may be related to the remodeling of the AV or the AA and LV remodeling secondary to the hemodynamic changes seen in patients with AR; this may impose a state of enhanced oxidative stress.

\section{Methods}

\section{Patients}

Thirty-one patients with moderate to severe or severe AR who were followed in the Valvular Disease Clinic in the Kaplan Medical Center were included in the study. Patients with AR were compared to 30 consecutive patients of a similar age, gender and atherosclerosis risk factor profile with no significant valvular disease. Patients with aortic stenosis (i.e. an AV area $\leq 1.5 \mathrm{~cm}^{2}$ ), mitral stenosis and more than mild mitral regurgitation were excluded. $\mathrm{Pa}$ tients with a history of acute coronary syndrome or revascularization in the previous 3 months, or any type of malignant or hematologic disorder were also excluded. This study was approved by the institutional ethics committee and all patients provided their written informed consent.

\section{Assessment of Risk Factors}

Risk factors were assessed for all the patients based on medical records. We defined diabetes mellitus as hyperglycemia requiring pharmacologic therapy, hypertension as either a systolic or diastolic increase in blood pressure $(>140 / 90 \mathrm{~mm} \mathrm{Hg})$ or the use of an antihypertensive therapy, hypercholesterolemia as a total cholesterol level $>200 \mathrm{mg} / \mathrm{dl}$ or the use of lipid-lowering agents and cigarette smoking as being an active smoker or having a smoking history of at least 10 pack-years. CAD was defined as a history of myocardial infarction or the presence of CAD on coronary angiography.

\section{Echocardiography Studies}

Transthoracic echocardiography including assessment of the $\mathrm{AV}$ was performed according to established guidelines [19]. LV volumes and LV ejection fraction (LVEF) were assessed by the modified Simpson's method [20]. The AR vena contracta, the pressure half-time, the jet density, the diastolic flow reversal in the descending aorta and the regurgitant fraction were used for assessment of AR severity [21]. Aortic root dimension and AA diameter were assessed according to the established guidelines [20].

\section{Anti-Oxidized LDL Antibodies}

Ninety-six well polystyrene plates (Nunc Maxisorp, Denmark) were coated with either copper oxLDL, native LDL (at a concentration of $10 \mu \mathrm{g} / \mathrm{ml}$ in PBS) or PBS overnight at $4^{\circ} \mathrm{C}$ as described [22]. After washing 4 times with PBS containing $0.05 \%$ Tween and $0.001 \%$ aprotinin (Sigma, USA), the plates were blocked with $2 \%$ BSA for $2 \mathrm{~h}$ at room temperature. Serum samples were diluted to 1:50 in PBS containing $0.05 \%$ Tween and $0.2 \%$ BSA. After additional overnight incubation, the sera were washed and alkaline-phosphatase-conjugated goat anti-human IgG (Jackson ImmunoResearch Laboratory Inc., USA) was added diluted 1:10,000 in PBS containing $0.05 \%$ 
Tween and $0.2 \%$ BSA for $1 \mathrm{~h}$ at room temperature. After extensive washing, $1 \mathrm{mg} / \mathrm{ml} \mathrm{p}$-nitrophenyl-phosphate (Sigma) in $50 \mathrm{~mm}$ carbonate buffer containing $1 \mathrm{mM} \mathrm{MgCl}_{2}$ ( $\mathrm{pH}$ 9.8) was added as a substrate. The reaction was stopped after $30 \mathrm{~min}$ by adding $1 \mathrm{M}$ of $\mathrm{NaOH}$. The color was read at a $405-\mathrm{nm}$ wavelength in a Titertek ELISA reader (S.L.T. Laboratory Instruments, Vienna, Austria) and results were expressed as an optical density of $405 \mathrm{~nm}$.

\section{C-Reactive Protein Analysis}

The serum levels of high-sensitivity C-reactive protein (hsCRP) were measured with the CardioPhase hsCRP reagent (Siemens Healthcare Diagnostics) according to the manufacturer's instructions.

\section{Statistical Analysis}

Continuous data are presented as medians and interquartile ranges (25th-75th percentiles) for skewed distributed variables or as a mean $\pm \mathrm{SD}$ when normally distributed. Categorical data are presented as absolute numbers and respective percentages. For categorical variables, the $\chi^{2}$ test was used. The Student $t$ test was used for continuous variables and the One-way Anova test was used to compare anti-oxLDL Abs in patients with different AR etiologies. Correlation analyses between anti-oxLDL Abs and various parameters were provided using Pearson correlation coefficients. Regression analysis and analysis of variance for anti-oxLDL Abs by independent variables was performed. Multilinear regression analyses were tested using a stepwise backward selection of the explicative variables. Although we made multiple comparisons, we did not perform multiple comparison correction because this was an observational study. All the above analyses were considered significant at $\mathrm{p} \leq 0.05$.

\section{Results}

The study included 31 consecutive patients with moderate-to-severe and severe AR and a control group of 30 patients who met the inclusion criteria. The etiology of AR was degenerative disease in 18 patients (58\%), bicuspid AV in $10(32 \%)$, postendocarditis in $2(6 \%)$ and rheumatic fever in 1 (3\%). Five patients (17\%) had known CAD, $1(3 \%)$ had a history of peripheral vascular disease and 1 had carotid stenosis. Six patients (19\%) had paroxysmal or chronic atrial fibrillation. The mean NYHA functional class of the patients was $1.7 \pm 0.8$. The control group included 18 patients (60\%) with stable chest pain who were referred for coronary angiography based on clinical indications and $12(40 \%)$ with a history of atrial arrhythmia and no valvular disease. Ten of the 18 patients with chest pain (33\% of the entire control group) had CAD on angiography. On transthoracic echocardiography, 14 patients had a dilated AA ( $>39 \mathrm{~mm}): 11$ with AR and 3 with no AR.

Baseline characteristics and laboratory results of the patients are shown in table 1 . There was no significant dif-
Table 1. Baseline characteristics of the studied patients

\begin{tabular}{lccl}
\hline & $\begin{array}{l}\text { AR } \\
(\mathrm{n}=31)\end{array}$ & $\begin{array}{c}\text { Controls } \\
(\mathrm{n}=30)\end{array}$ & $\mathrm{p}$ \\
\hline Age, years & $62 \pm 12$ & $66 \pm 13$ & 0.11 \\
Male & $23(74)$ & $24(80)$ & 0.81 \\
Hypertension & $19(61)$ & $20(67)$ & 0.87 \\
Diabetes mellitus & $11(35)$ & $5(17)$ & 0.12 \\
Hypercholesterolemia & $20(64)$ & $18(60)$ & 0.92 \\
Smoking & $10(32)$ & $5(17)$ & 0.26 \\
CAD/peripheral artery disease & $7(23)$ & $10(33)$ & 0.52 \\
Atrial fibrillation & $6(19)$ & $12(40)$ & 0.14 \\
Aspirin therapy & $19(61)$ & $17(57)$ & 0.87 \\
Statin therapy & $19(61)$ & $18(60)$ & 0.91 \\
Beta-blockers & $21(68)$ & $18(60)$ & 0.59 \\
ACE inhibitors & $23(74)$ & $15(50)$ & 0.09 \\
Hb, g/dl & $13.5 \pm 1.5$ & $13.8 \pm 1.6$ & 0.2 \\
WBC count & $6.7 \pm 1.5$ & $7.6 \pm 2.5$ & 0.046 \\
Platelets & $211 \pm 39$ & $231 \pm 95$ & 0.2 \\
Creatinine, mg/dl & $1.09 \pm 0.22$ & $0.96 \pm 0.18$ & 0.009 \\
Total cholesterol, mg/dl & $164.3 \pm 25$ & $169.1 \pm 33$ & 0.3 \\
LDL cholesterol, mg/dl & $92 \pm 24$ & $97 \pm 27$ & 0.25 \\
Triglycerides, mg/dl & $115.7 \pm 43$ & $121 \pm 49$ & 0.31 \\
HDL cholesterol, mg/dl & $48 \pm 12$ & $52 \pm 17$ & 0.21 \\
CRP, mg/dl & $1.7 \pm 1.6$ & $2.1 \pm 2$ & 0.48 \\
\hline
\end{tabular}

Figures in parentheses are percentages. $\mathrm{ACE}=$ Angiotensinconverting enzyme.

Table 2. Echocardiographic parameters in patients with $A R$ and controls

\begin{tabular}{lcrc}
\hline & $\begin{array}{l}\text { AR } \\
(\mathrm{n}=31)\end{array}$ & $\begin{array}{r}\text { Control } \\
(\mathrm{n}=30)\end{array}$ & $\mathrm{p}$ \\
\hline LV end-diastolic diameter, mm & $54 \pm 4.9$ & $47 \pm 4.8$ & $<0.0001$ \\
LV end-systolic diameter, mm & $34.6 \pm 7.3$ & $29.6 \pm 5.2$ & 0.01 \\
Septal thickness, mm & $12.3 \pm 1$ & $11 \pm 2.3$ & 0.01 \\
Posterior wall thickness, mm & $11.5 \pm 1.7$ & $10 \pm 1.9$ & 0.01 \\
LVEF, \% & $57 \pm 8.2$ & $53 \pm 8.5$ & 0.07 \\
Aortic root diameter, mm & $35 \pm 5.2$ & $30 \pm 3.5$ & $<0.001$ \\
AA diameter, mm & $37 \pm 4.9$ & $35 \pm 4.9$ & 0.005 \\
Left atrial area, $\mathrm{cm}^{2}$ & $23 \pm 4.7$ & $21 \pm 4.2$ & 0.08 \\
\hline
\end{tabular}

ference in the prevalence of CAD between patients with $\mathrm{AR}$ and the control group. There were no significant differences in CRP levels, hemoglobin ( $\mathrm{Hb}$ ), lipid profile and lipid-lowering treatment or aspirin treatment between the groups. However, patients with $\mathrm{AR}$ had higher creatinine levels and a lower white blood cell (WBC) count.

Patients with AR had a greater LV diameter and septal and posterior wall thickness (table 2). The diameters of 


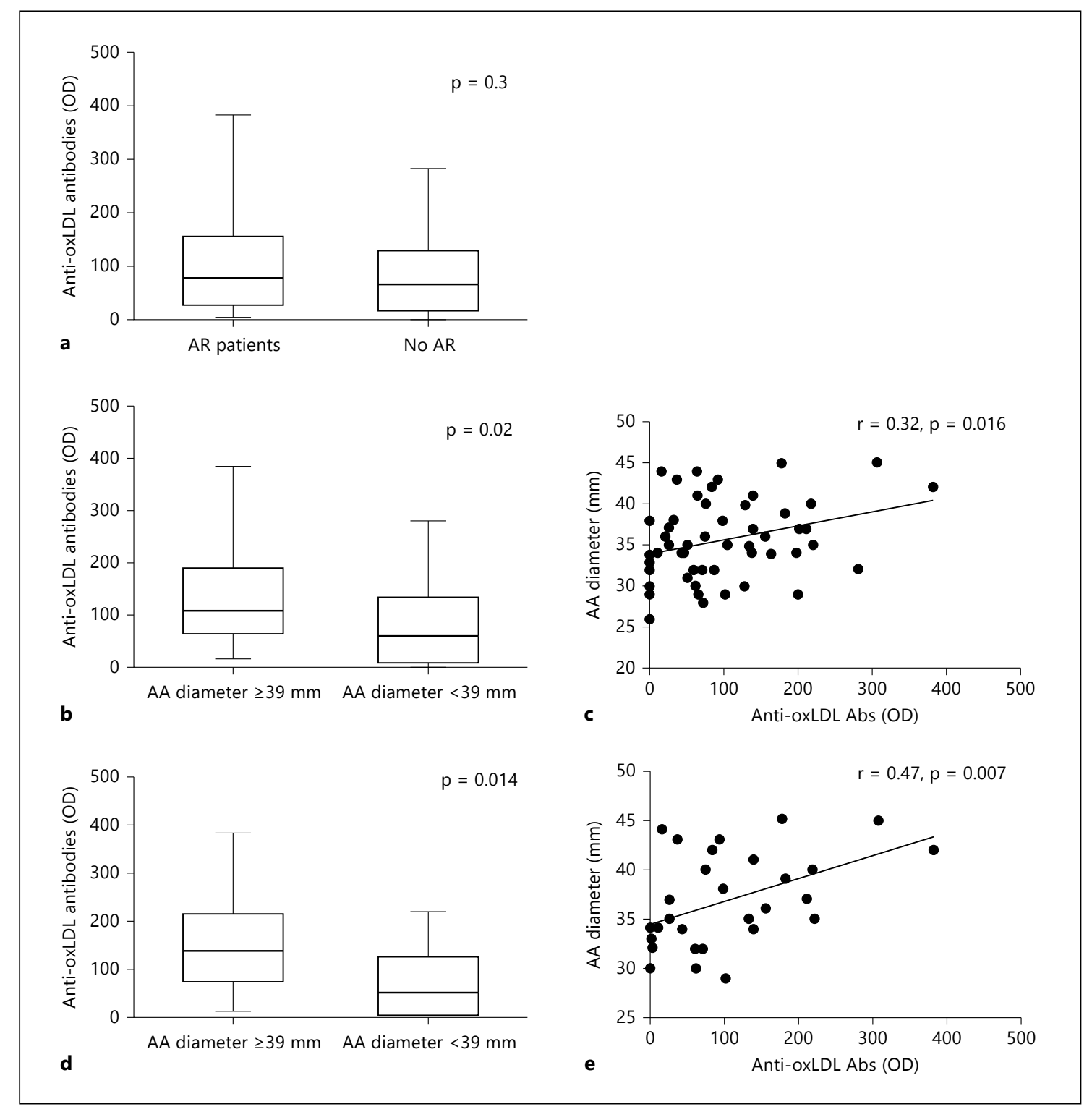

Fig. 1. a oxLDL Abs in patients with and without AR. b Correlation between oxLDL Abs and AA diameter. c oxLDL Abs in patients with and without AA dilatation. d Correlation between oxLDL Abs and AA diameter in patients with AR. e oxLDL Abs in patients with and without AA dilatation in patients with AR.

the aortic root and the AA were higher in patients with AR. The AR mean vena contracta area was $6.1 \pm 0.14 \mathrm{~mm}$.

There was no difference in the level of anti-oxLDL Abs between patients with AR and patients with no AR (100 \pm 17 vs. $77 \pm 13$ units, $p=0.3$; fig. 1a). There was also no difference in oxLDL Ab levels among patients with different AR etiologies $(p=0.43)$. However, in both study groups, anti-oxLDL Abs correlated positively with AA diameter ( $\mathrm{r}=0.32, \mathrm{p}=0.016$; fig. $1 \mathrm{c})$, and the level of oxLDL Abs was significantly higher in patients with an
AA diameter $\geq 39 \mathrm{~mm}(141 \pm 28$ vs. $79 \pm 12$ units, $\mathrm{p}=0.02$; fig. 1b). Tables 3 and 4 present the baseline clinical and echocardiographic parameters of patients with and without dilatation of the AA. The baseline parameters of the 2 groups did not differ significantly, except for in LV hypertrophy, seen in patients with a dilated AA.

Anti-oxLDL Abs also correlated positively with WBC count $(\mathrm{r}=0.32, \mathrm{p}=0.015)$ and creatinine level $(\mathrm{r}=0.27$, $\mathrm{p}=0.04)$ and a negative association was observed between anti-oxLDL Abs and diabetes mellitus $(r=-0.28, \mathrm{p}=$ 
0.03). On multivariate analysis, only AA diameter and WBC count were related to oxLDL Abs level in all patients (table 5).

Figure $1(\mathrm{~d}, \mathrm{e})$ shows that the correlation between oxLDL Abs and AA diameter became even more robust when only patients with AR were considered $(r=0.47$ and $\mathrm{p}=0.007)$ and a significant difference was evident between patients with AR that had a dilated AA and those with no dilatation ( $155 \pm 34$ vs. $69 \pm 16$ units, $\mathrm{p}=0.0014)$. In patients with $\mathrm{AR}$, oxLDL Abs correlated positively with the size of the AA and a negative association was seen in the presence of diabetes (table 5).

\section{Discussion}

We undertook this study to evaluate the levels of oxLDL Abs in patients with AV regurgitation. In previous reports, oxLDL Abs were shown to be related to the progression and prognosis of congestive heart failure [23, 24]. Volume overload in significant AR causes progressive LV remodeling characterized by chamber dilation and eccentric cardiac myocyte hypertrophy [25]. This process is regulated by mechanical stress, neurohormonal activation, inflammation and oxidative stress $[26,27]$. Since oxLDL Abs are a measurable marker of oxidative stress [9], we hypothesized that the levels of oxLDL Abs in patients with significant AR would increase. In addition, oxLDL Abs, as an oxidative stress marker, have been seen to have accumulated on semilunar valves in patients with atherosclerosis, suggesting this to be an additional mechanism accelerating valvular degeneration [28] and potentially triggering the production of oxLDL Abs. The results of our study, however, did not show a difference between the patients with AR and the controls in the oxLDL Ab levels. There are several possible explanations for this finding. First, our control group also had risk factors and there were more patients with CAD in the control group. Another important point is that the majority $(85 \%)$ of the patients in our AR population was in the functional class 1 or 2 with no history of recurrent hospitalization and the increase in the titer of oxLDL Abs is seen in patients with advanced heart failure. An additional potential explanation is a reduction in circulating oxLDL Abs due to the binding of oxLDL present in the semilunar valves where oxLDL had been detected, thus forming immune complexes.

We showed that oxLDL Abs correlated positively with AA diameter, WBC count and creatinine level. A negative association was observed with diabetes mellitus. When only patients with AR were included, the association be-
Table 3. Comparison of baseline characteristics in patients with and without dilatation of the AA

\begin{tabular}{lccl}
\hline & $\begin{array}{c}\mathrm{AA} \geq 39 \mathrm{~mm} \\
(\mathrm{n}=14)\end{array}$ & $\begin{array}{l}\mathrm{AA}<39 \mathrm{~mm} \\
(\mathrm{n}=47)\end{array}$ & $\mathrm{p}$ \\
\hline Age, years & $66 \pm 10$ & $64 \pm 13$ & 0.3 \\
Male & $12(86)$ & $35(74)$ & 0.5 \\
Hypertension & $9(64)$ & $30(64)$ & 0.8 \\
Diabetes mellitus & $2(14)$ & $14(30)$ & 0.3 \\
Hypercholesterolemia & $10(71)$ & $28(60)$ & 0.6 \\
Smoking & $3(21)$ & $12(30)$ & 1 \\
CAD/peripheral artery disease & $4(28)$ & $13(28)$ & 1 \\
Atrial fibrillation & $3(21)$ & $15(32)$ & 0.5 \\
Aspirin therapy & $8(57)$ & $28(60)$ & 0.9 \\
Statin therapy & $7(50)$ & $23(49)$ & 0.8 \\
Beta-blockers & $8(57)$ & $31(66)$ & 0.8 \\
ACE inhibitors & $11(78)$ & $27(57)$ & 0.3 \\
Hb, g/dl & $13.6 \pm 1$ & $13.7 \pm 1$ & 0.4 \\
WBC count & $6.9 \pm 1.6$ & $6.4 \pm 1.3$ & 0.5 \\
Platelets & $211 \pm 35$ & $208 \pm 40$ & 0.7 \\
Creatinine, mg/dl & $1.1 \pm 0.2$ & $1.0 \pm 0.2$ & 0.1 \\
Total cholesterol, mg/dl & $154 \pm 30$ & $170 \pm 28$ & 0.09 \\
LDL cholesterol, mg/dl & $91 \pm 27$ & $95 \pm 25$ & 0.6 \\
Triglycerides, mg/dl & $104 \pm 44$ & $120 \pm 47$ & 0.3 \\
HDL cholesterol, mg/dl & $46 \pm 14$ & $52 \pm 13$ & 0.2 \\
CRP, mg/dl & $2.5 \pm 3$ & $1.8 \pm 1.4$ & 0.2 \\
\hline
\end{tabular}

Figures in parentheses are percentages. ACE $=$ Angiotensin-converting enzyme.

Table 4. Echocardiographic parameters in patients with and without dilatation of the AA

\begin{tabular}{lccc}
\hline & $\begin{array}{l}\mathrm{AA} \geq 39 \mathrm{~mm} \\
(\mathrm{n}=14)\end{array}$ & $\begin{array}{l}\mathrm{AA}<39 \mathrm{~mm} \\
(\mathrm{n}=47)\end{array}$ & $\mathrm{p}$ \\
\hline LV end-diastolic diameter, $\mathrm{mm}$ & $52.6 \pm 6.3$ & $50 \pm 6$ & 0.17 \\
LV end-systolic diameter, mm & $34.4 \pm 0.4$ & $31 \pm 6.5$ & 0.16 \\
Septal thickness, mm & $12.3 \pm 1.3$ & $11.4 \pm 1.6$ & 0.08 \\
Posterior wall thickness, mm & $11.6 \pm 1.7$ & $10.1 \pm 1.5$ & 0.002 \\
LVEF, \% & $58 \pm 3$ & $55.3 \pm 1.4$ & 0.3 \\
Aortic root diameter, mm & $36.7 \pm 4$ & $32.4 \pm 3.8$ & 0.004 \\
AA diameter, mm & $42.1 \pm 2$ & $33.3 \pm 3$ & $<0.001$ \\
Left atrial area, $\mathrm{cm}{ }^{2}$ & $23.9 \pm 3.4$ & $21.3 \pm 4.6$ & 0.11 \\
\hline
\end{tabular}

tween oxLDL Abs and AA diameter was even more pronounced. Although AR patients with a dilated AA may represent a group of patients with more advanced disease, we did not observe a difference in functional class in patients with aortic dilatation and those with no aortic dilatation. The hemodynamic forces in the AA may, however, not be related to the functional class. 
Table 5. Association of IgG oxLDL Ab levels with baseline clinical characteristics in patients with AR

\begin{tabular}{|c|c|c|c|c|c|c|c|c|}
\hline & \multicolumn{4}{|c|}{ All patients } & \multicolumn{4}{|c|}{ Patients with AR } \\
\hline & \multicolumn{2}{|c|}{ univariate analysis } & \multicolumn{2}{|c|}{ multivariate analysis } & \multicolumn{2}{|c|}{ univariate analysis } & \multicolumn{2}{|c|}{ multivariate analysis } \\
\hline & $\mathrm{R}$ & $\mathrm{p}$ & Std. coeff. & $\mathrm{p}$ & $\mathrm{R}$ & $\mathrm{p}$ & Std. coeff. & $\mathrm{p}$ \\
\hline AA diameter, $\mathrm{mm}$ & 0.32 & 0.016 & 0.346 & 0.009 & 0.47 & 0.007 & 0.473 & 0.007 \\
\hline WBC count & 0.32 & 0.015 & 0.344 & 0.009 & 0.24 & 0.2 & & \\
\hline Creatinine, mg/dl & 0.27 & 0.04 & & & 0.2 & 0.29 & & \\
\hline Diabetes & -0.28 & 0.03 & & & -0.43 & 0.014 & & 0.082 \\
\hline
\end{tabular}

Age, sex, smoking, hyperlipidemia, hypertension, ischemic heart disease, Hb, platelets, total cholesterol, triglycerides, LDL cholesterol, HDL cholesterol, medications, LVEF, LV end-diastolic diameter, LV end-systolic diameter and the thickness of the septal and posterior walls were not associated with levels of IgG anti-oxLDL Abs. Std. coeff. $=$ Standardized coefficient.

To the best of our knowledge, there are no previous reports on oxLDL Abs in patients with AA dilatation. Hobbs et al. [29] demonstrated a significant association between plasma LDL levels and the presence of small abdominal aortic aneurysms (AAAs). Chronic inflammation is a prominent characteristic of AAAs, in which the outer wall is infiltrated by lymphocytes and macrophages [30]. The authors suggested a hypothesis that LDL, in its oxidized form, possibly acting via inflammation-mediated matrix degeneration, could be an initiating factor in the development of aortic aneurysms.

However, Lindholt et al. [31] assessed the role of risk factors and oxLDL Abs in patients with expanding AAAs. They did not find a correlation between lipids/oxLDL Abs and AAA expansion.

In a recent study, van Bommel et al. [32] showed no significant difference between patients with stable chronic periaortitis and control subjects with regard to oxLDL Ab titers. Our study population consisted of patients with AA dilatation due to AR or hypertension, and none of the patients had AAA. We also did not find a correlation between LDL cholesterol and AA dilatation in any of the patients; we did not measure oxLDL, however.

Thoracic aortic aneurysm is a chronic inflammatory disease. Although numerous studies have been conducted to clarify the pathogenesis of this disease and the mechanisms involved, the latter have still to be determined. Several potential mechanisms have been proposed, including increased hemodynamic forces, transmural inflammation and destructive-remodeling of the extracellular matrix [33]. An imbalance between matrix metalloproteinases and their inhibitors is crucial in the remodeling of the aortic wall, and the enzymatic activities of these factors are more enhanced by inflammatory cell infiltrates $[34,35]$. Reactive oxygen species activate matrix metalloproteinases, suggesting a possible role of oxidative stress in the pathogenesis of aortic aneurysms [36]. This finding is supported by Ejiri et al. [37], whose study demonstrated the role of 22 phox-based NADH/NADPH oxidase and the local renin-angiotensin system in the pathogenesis of thoracic aortic aneurysms.

AA dilatation in patients with AR is multifactorial, and may be due to intrinsic changes in the aortic wall, hemodynamic changes or both. The interaction between $\mathrm{AV}$ and aortic wall pathology is currently not clear. Aortic wall pathology with aneurysm formation is frequently associated with a structural deterioration of the AV. Dilatation of the aortic root and the AA is a known cause of $\mathrm{AV}$ regurgitation due to commissural displacement and malcoaptation of the aortic cusps. A 3-dimensional model showed that dilatation of the aortic root causes increased leaflet stress and strain [38]. Thickening of leaflet freemargins was described in patients with aortic root dilatation and AR [39]. oxLDL levels have been shown to be related to AV remodeling in aortic stenosis [3]; it is possible that in patients with $\mathrm{AR}$, oxLDL is also related to leaflet remodeling.

The dilatation of the aortic root and the AA in patients with AR may also be caused or worsened by the hemodynamic changes associated with AR. These are even seen in patients with a 'normally' functioning bicuspid $\mathrm{AV}$; it appears that, in addition to genetic factors, the AA dilatation they experience is partly due to eccentric flow [40]. This qualitatively altered flow, which affects the bicuspid AV individual from a very young age, does not meet the standard criteria for aortic stenosis by common echocardiographic methods of grading and can only be characterized with sophisticated 4-dimensional flow 
magnetic resonance imaging [41]. Moreover, a significant correlation has recently been demonstrated between the degree of eccentricity of the systolic transvalvular flow and the severity of the proximal aortic dilatation in the pediatric bicuspid AV population [42]. These abnormal flow patterns act over long periods of time and may lead to asymmetric, stress-induced aortic wall lesions and subsequent remodeling and dilatation of specific aortic segments, even in the absence of echocardiographically significant valvular lesions $[40,43,44]$. These hemodynamic changes may cause the local inflammation and increased oxidative stress that were shown to be related to the production of oxLDL Abs. Our results, which show that patients with wider AAs and thus a higher turbulent flow with increased shear stress on the $\mathrm{AV}$ and AV leaflets have increased levels of oxLDL Abs, support this concept.

We found diabetes mellitus, a known risk factor, to be negatively associated with oxLDL Abs. This negative association has been described previously [45]; however, immune complexes containing apolipoprotein B were shown to be significantly higher in patients with diabetes than in controls. Together, these findings might reflect a higher affinity of oxLDL $\mathrm{Ab}$ in diabetes patients that is likely to increase the generation of oxLDL, anti-oxLDL immune complexes and reduce free oxLDL Abs. Although we did not observe an inverse correlation between diabetes and aortic aneurysm, previous epidemiological investigations indicate that diabetes may be a negative risk factor for aortic aneurysm formation, although the precise mechanisms here remain undetermined [46].

Our study had some limitations, the main one being the small population size. We included only patients with 'pure' AR, which is a somewhat less-common valvular disease. In order to explore these findings, a multicenter study needs to be designed. The second limitation is that the anti-oxLDL Abs ELISA method is not a standardized assay and cross-sectional comparative studies cannot be optimally conducted. Another is the difficulty to homogenize the population with regard to atherosclerosis and inflammation. Although the prevalence of coronary artery and peripheral vascular diseases was similar in both groups, we did not perform other assessments such as carotid artery intima-media thickness. There were more patients with atrial fibrillation in the control group compared to the AR group, although this difference was not statistically significant. In order to homogenize the groups with regard to the inflammatory burden, we performed hs-CRP, which was similar in the 2 groups.

\section{Conclusion}

We did not find a difference in oxLDL Ab levels between patients with AR and controls; however, there was a strong correlation between oxLDL Abs and AA diameter, with significantly elevated levels in patients in whom this diameter was $>39 \mathrm{~mm}$.

\section{References}

1 Holvoet P, Vanhaecke J, Janssens S, Van de Werf F, Collen D: Oxidized LDL and malondialdehyde-modified LDL in patients with acute coronary syndromes and stable coronary artery disease. Circulation 1998;98:1487-1494.

2 Holvoet P, Mertens A, Verhamme P, et al: Circulating oxidized LDL is a useful marker for identifying patients with coronary artery disease. Arterioscler Thromb Vasc Biol 2001; 21:844-848.

3 Cote C, Pibarot P, Despres JP, et al: Association between circulating oxidised low-density lipoprotein and fibrocalcific remodelling of the aortic valve in aortic stenosis. Heart 2008; 94:1175-1180.

-4 Toshima S, Hasegawa A, Kurabayashi M, Itabe H, Takano T, Sugano J, Shimamura K, Kimura J, Michishita I, Suzuki T, et al: Circulating oxidized low density lipoprotein levels. A biochemical risk marker for coronary heart disease. Arterioscler Thromb Vasc Biol 2000; 20:2243-2247.
5 Inoue T, Uchida T, Kamishirado H, Takayanagi K, Hayashi T, Morooka S: Clinical significance of antibody against oxidized low density lipoprotein in patients with therosclerotic coronary artery disease. J Am Coll Cardiol 2001;37:775-779.

6 Chen Q, Reis SE, Kammerer C, Craig W, McNamara DM, Holubkov R, Sharaf BL, Sopko G, Pauly DF, Merz CN, et al: Association of anti-oxidized LDL and candidate genes with severity of coronary stenosis in the Women's Ischemia Syndrome Evaluation study. J Lipid Res 2011;52:801-807.

7 Shetty R, Pibarot P, Audet A, et al: Lipid-mediated inflammation and degeneration of bioprosthetic heart valves. Eur J Clin Invest 2009; 39:471-480.

$>8$ Witztum JL, Horkko S: The role of oxidized LDL in atherogenesis: immunological response and anti-phospholipid antibodies. Ann NY Acad Sci 1997;811:88-96.
Steinerova A, Racek J, Stozicky F, Zima T, Fialova L, Lapin A: Antibodies against oxidized LDL - theory and clinical use. Physiol Res 2001;50:131-141.

10 Lehtimaki T, Lehtinen S, Solakivi T, et al: Autoantibodies against oxidized low density lipoprotein in patients with angiographically verified coronary artery disease. Arterioscler Thromb Vasc Biol 1999;19:23-27.

11 Salonen JT, Yla-Herttuala S, Yamamoto R, et al: Autoantibody against oxidised LDL and progression of carotid atherosclerosis. Lancet 1992;339:883-887.

12 Andican G, Seven A, Uncu M, Cantasdemir M, Numan F, Burcak G: Oxidized LDL and anti-oxLDL antibody levels in peripheral atherosclerotic disease. Scand J Clin Lab Invest 2008;68:473-478.

13 Puurunen M, Manttari M, Manninen V, et al: Antibody against oxidized low-density lipoprotein predicting myocardial infarction. Arch Intern Med 1994;154:2605-2609. 
14 Bekeredjian R, Grayburn PA: Valvular heart disease: aortic regurgitation. Circulation 2005;112:125-134.

-15 Kervancioglu S, Davutoglu V, Ozkur A, Sezen Y, Kervancioglu R, Bayram MM: Carotid and brachial intima-media thickness, arterial diameter and resistivity indices in aortic regurgitation. Acta Radiol 2004;45:815-818.

$\checkmark 16$ Su TC, Chien KL, Jeng JS, et al: Pulse pressure, aortic regurgitation and carotid atherosclerosis: a comparison between hypertensives and normotensives. Int J Clin Pract 2006;60:134140.

17 Shimoni S, Zilberman L, Edri O, et al: Thoracic aortic atherosclerosis in patients with aortic regurgitation. Atherosclerosis 2011; 218:107-109.

18 Shimoni S, Bar I, Zilberman L, et al: Circulating progenitor and apoptotic progenitor cells in patients with aortic regurgitation. Circ J 2013;77:764-771.

$\checkmark 19$ Bonow RO, Carabello BA, Chatterjee K, et al: 2008 Focused update incorporated into the ACC/AHA 2006 guidelines for the management of patients with valvular heart disease: a report of the American College of Cardiology/American Heart Association Task Force on Practice Guidelines (Writing Committee to Revise the 1998 Guidelines for the Management of Patients with Valvular Heart Disease): endorsed by the Society of Cardiovascular Anesthesiologists, Society for Cardiovascular Angiography and Interventions and Society of Thoracic Surgeons. Circulation 2008;118:e523-e661.

20 Lang RM, Bierig M, Devereux RB, et al: Recommendations for chamber quantification: a report from the American Society of Echocardiography's Guidelines and Standards Committee and the Chamber Quantification Writing Group, developed in conjunction with the European Association of Echocardiography, a branch of the European Society of Cardiology. J Am Soc Echocardiogr 2005; 18:1440-1463.

-21 Zoghbi WA, Enriquez-Sarano M, Foster E, et al: Recommendations for evaluation of the severity of native valvular regurgitation with two-dimensional and Doppler echocardiography. J Am Soc Echocardiogr 2003;16:777802.

22 George J, Harats D, Bakshi E, et al: Anti-oxidized low density lipoprotein antibody determination as a predictor of restenosis following percutaneous transluminal coronary angioplasty. Immunol Lett 1999;68:263-266.

$\checkmark 23$ George J, Wexler D, Roth A, Barak T, Sheps D, Keren G: Usefulness of anti-oxidized LDL antibody determination for assessment of clinical control in patients with heart failure. Eur J Heart Fail 2006;8:58-62.
24 Charach G, George J, Afek A, et al: Antibodies to oxidized LDL as predictors of morbidity and mortality in patients with chronic heart failure. J Card Fail 2009;15:770-774.

25 Plante E, Couet J, Gaudreau M, Dumas MP, Drolet MC, Arsenault M: Left ventricular response to sustained volume overload from chronic aortic valve regurgitation in rats. J Card Fail 2003;9:128-140.

26 Ryan TD, Rothstein EC, Aban I, et al: Left ventricular eccentric remodeling and matrix loss are mediated by bradykinin and precede cardiomyocyte elongation in rats with volume overload. J Am Coll Cardiol 2007;49:811-821.

27 Borer JS, Herrold EM, Carter JN, Catanzaro DF, Supino PG: Cellular and molecular basis of remodeling in valvular heart diseases. Heart Fail Clin 2006;2:415-424.

28 Mehrabi MR, Sinzinger H, Ekmekcioglu C, et al: Accumulation of oxidized LDL in human semilunar valves correlates with coronary atherosclerosis. Cardiovasc Res 2000;45:874882.

29 Hobbs SD, Claridge MW, Quick CR, Day NE, Bradbury AW, Wilmink AB: LDL cholesterol is associated with small abdominal aortic aneurysms. Eur J Vasc Endovasc Surg 2003;26: 618-622.

30 Brophy CM, Reilly JM, Smith GJ, Tilson MD: The role of inflammation in nonspecific abdominal aortic aneurysm disease. Ann Vasc Surg 1991;5:229-233.

31 Lindholt JS, Heegaard NH, Vammen S, Fasting $\mathrm{H}$, Henneberg EW, Heickendorff L: Smoking, but not lipids, lipoprotein(a) and antibodies against oxidised LDL, is correlated to the expansion of abdominal aortic aneurysms. Eur J Vasc Endovasc Surg 2001;21:5156.

32 van Bommel EF, van Tits LJ, van den Berg EA, Prins J, Stalenhoef AF: Autoantibodies against oxidized low-density lipoprotein and lipid profile in patients with chronic periaortitis: case-control study. Rheumatol Int 2011;31: 201-208.

33 Patel MI, Hardman DT, Fisher CM, Appleberg M: Current views on the pathogenesis of abdominal aortic aneurysms. J Am Coll Surg 1995; 181:371-382.

-34 Freestone T, Turner RJ, Coady A, Higman DJ, Greenhalgh RM, Powell JT: Inflammation and matrix metalloproteinases in the enlarging abdominal aortic aneurysm. Arterioscler Thromb Vasc Biol 1995; 15:1145-1151.

35 Knox JB, Sukhova GK, Whittemore AD, Libby P: Evidence for altered balance between matrix metalloproteinases and their inhibitors in human aortic diseases. Circulation 1997;95:205-212.
36 Siwik DA, Pagano PJ, Colucci WS: Oxidative stress regulates collagen synthesis and matrix metalloproteinase activity in cardiac fibroblasts. Am J Physiol Cell Physiol 2001;280: C53-C60.

37 Ejiri J, Inoue N, Tsukube $\mathrm{T}$, et al: Oxidative stress in the pathogenesis of thoracic aortic aneurysm: protective role of statin and angiotensin II type 1 receptor blocker. Cardiovasc Res 2003;59:988-996.

38 Grande KJ, Cochran RP, Reinhall PG, Kunzelman KS: Mechanisms of aortic valve incompetence: finite element modeling of aortic root dilatation. Ann Thorac Surg 2000;69: 1851-1857.

39 Turri M, Thiene G, Bortolotti U, Milano A, Mazzucco A, Gallucci V: Surgical pathology of aortic valve disease. A study based on 602 specimens. Eur J Cardiothorac Surg 1990;4: 556-560.

40 Conti CA, Della Corte A, Votta E, et al: Biomechanical implications of the congenital bicuspid aortic valve: a finite element study of aortic root function from in vivo data. J Thorac Cardiovasc Surg 2010;140:890-896, $6 \mathrm{e} 1-2$.

41 Hope MD, Meadows AK, Hope TA, et al: Images in cardiovascular medicine. Evaluation of bicuspid aortic valve and aortic coarctation with 4D flow magnetic resonance imaging. Circulation 2008;117:2818-2819.

42 den Reijer PM, Sallee D 3rd, van der Velden $P$, et al: Hemodynamic predictors of aortic dilatation in bicuspid aortic valve by velocityencoded cardiovascular magnetic resonance. J Cardiovasc Magn Res 2010;12:4.

43 Della Corte A, Quarto C, Bancone C, et al: Spatiotemporal patterns of smooth muscle cell changes in ascending aortic dilatation with bicuspid and tricuspid aortic valve stenosis: focus on cell-matrix signaling. J Thorac Cardiovasc Surg 2008;135:8-18, e1-2.

44 Lehoux S, Tronc F, Tedgui A: Mechanisms of blood flow-induced vascular enlargement. Biorheology 2002;39:319-324.

45 Orchard TJ, Virella G, Forrest KY, Evans RW, Becker DJ, Lopes-Virella MF: Antibodies to oxidized LDL predict coronary artery disease in type 1 diabetes: a nested case-control study from the Pittsburgh Epidemiology of Diabetes Complications Study. Diabetes 1999;48: 1454-1458.

46 Lederle FA, Johnson GR, Wilson SE, et al: The aneurysm detection and management study screening program: validation cohort and final results. Aneurysm Detection and Management Veterans Affairs Cooperative Study Investigators. Arch Intern Med 2000;160: $1425-1430$
Anti-oxLDL Antibodies in Aortic

Regurgitation
Cardiology 2014;128:54-61 DOI: $10.1159 / 000357835$ 